\title{
Increased ethane exhalation, an in vivo index of lipid peroxidation, in alcohol-abusers
}

\author{
P Lettéron, V Duchatelle, A Berson, B Fromenty, C Fisch, C Degott, J P Benhamou, \\ D Pessayre
}

\begin{abstract}
Ethane exhalation was measured in $\mathbf{4 2}$ control subjects, 52 patients with various non-alcoholic liver diseases, and 89 alcohol abusers who had been admitted to hospital for alcohol withdrawal and assessment of liver disease (six with normal liver tests, 10 with steatosis with or without fibrosis, six with alcoholic hepatitis, 29 with cirrhosis, 34 with both cirrhosis and alcoholic hepatitis, and four with both cirrhosis and a hepatocellular carcinoma). Ethane exhalation was similar in control subjects and in patients with non-alcoholic liver diseases, but was five times higher in alcohol abusers. Ethane exhalation in alcohol abusers was significantly, but very weakly, correlated with the daily ethanol intake before hospital admission, and the histological score for steatosis, but not with the inflammation or alcoholic hepatitis scores. Ethane exhalation was inversely correlated with the duration of abstinence before the test In nine alcoholic patients, the exhalation of ethane was measured repeatedly, and showed slow improvement during abstinence. Ethane exhalation was significantly but weakly correlated with the Pugh's score in patients with alcoholic cirrhosis. It is concluded that the mean ethane exhalation is increased in alcohol abusers. One of the possible mechanisms may be the presence of oxidizable fat in the liver. The weak correlation with the Pugh's score is consistent with the contribution of many other factors in the progression to severe liver disease.

(Gut 1993; 34: 409-414)
\end{abstract}

Experimental data support a role of free radical mechanisms, including lipid peroxidation, in ethanol induced cellular injury in animals.' The question of whether lipid peroxidation occurs in alcoholic patients, however, and if it does of its possible mechanism(s) and importance in the development of liver lesions, remains largely unanswered at present. Hepatic levels of conjugated dienes (an early product of lipid peroxidation) were found to be higher in 16 patients with alcoholic liver disease than in eight patients with non-alcoholic liver disease. Hepatic thiobarbituric acid reactants (malondialdehyde and other products of lipid peroxidation) were higher in 13 heavy drinkers with fatty livers (studied after about seven days of abstinence) than in five non-drinkers with histologically normal livers. ${ }^{3}$ In another study, serum and hepatic thiobarbituric acid reactive substances were found to be slightly increased in patients with chronic liver diseases, but did not show any difference between patients with chronic alcoholic liver disease and patients with chronic non-alcoholic liver disease.

Peroxidation may also be assessed noninvasively by determining the exhalation of volatile hydrocarbons (pentane and ethane) in breath. ${ }^{5}$ Pentane and ethane are formed during the process of lipid peroxidation and are exhaled in breath (see Discussion). A difficulty with pentane is that it is extensively oxidised in the liver.$^{56}$ In patients with liver disease, therefore, an increased exhalation of pentane may mean either an increased production rate (peroxidation), or a decreased hepatic metabolism. ${ }^{5}$ In contrast, ethane undergoes little hepatic metabolism, so that its exhalation mainly reflects its formation rate. ${ }^{5}$ An increased exhalation of pentane, but not of ethane, was found in 15 patients with alcoholic cirrhosis compared with 21 healthy controls. ${ }^{7}$ The duration of abstinence before breath sampling, however, was not mentioned. ${ }^{?}$

Yet another method of assessing in vivo lipid peroxidation is the measurement of serum vitamin $\mathrm{E}$ concentrations (vitamin $\mathrm{E}$ is consumed during peroxidation). Serum vitamin E concentrations, however depend on several other factors, including diet, absorption of this fat soluble vitamin, and serum lipid values. ${ }^{8-10} \mathrm{~A}$ decreased serum vitamin $E$ concentration in chronic alcoholic patients has been found in two studies. ${ }^{112}$ In another study, however, the serum vitamin $\mathrm{E} /$ serum cholesterol ratio was found to be normal in 21 of 25 patients with chronic alcoholic liver disease. ${ }^{13}$

The reasons for the apparent discrepancies in tests of lipid peroxidation in alcoholic patients in the published reports are not completely understood. They may include the inconsistant occurrence of lipid peroxidation in alcoholics, the small numbers of patients studied, differences in the liver diseases studied, and failure to take account of the duration of abstinence before the various measurements.

In view of these conflicting data, we decided to reinvestigate this question by a non-invasive, reliable test (the exhalation of ethane) in a large series of patients admitted to hospital for both alcohol withdrawal (thus enabling correct appreciation of the duration of abstinence) and assessment of the liver disease. Results were compared with a large group of controls, and with a large series of patients with various nonalcoholic liver diseases. In an effort to understand better the possible mechanism of increased lipid peroxidation in alcoholic patients and its significance in the development of liver lesions, correlations were looked for between the exhalation of ethane and several clinical and histological parameters. 


\section{Methods}

\section{PATIENTS}

Three groups of subjects were included in this study, which took place between July 1989 and February 1991. The first group included 42 healthy volunteers recruited mainly from the laboratory team or clinical staff. This group included 22 men and 20 women, aged 22 to 67 years, with a mean (SD) age of $40(8)$ years.

The second group consisted of 52 patients with non-alcoholic liver diseases. These were 29 men and 23 women, aged 21 to 78 years with a mean (SD) age of 49 (16) years. This group included two patients with a transplanted liver; six patients with acute hepatitis (two viral, four drug induced); 11 patients with chronic hepatitis (five because of hepatitis B virus, four hepatitis C virus, and two autoimmune); 17 patients with viral cirrhosis (six caused by hepatitis B virus, and 11 by hepatitis $C$ virus); one patient with polyadenomatosis of the liver; five patients with non-alcoholic hepatocellular carcinoma; two patients with liver metastases; five patients with sclerosing cholangitis; one with primary biliary cirrhosis; and two with extrahepatic bile duct obstruction.

The third group included 89 alcohol abusers admitted to hospital for both alcohol withdrawal and assessment of the liver disease (45 men, 44 women), aged 23 to 78 years, with a mean age (SD) of 49 (12) years. This group included six patients with normal hepatic tests, 10 patients with hepatic steatosis associated or not with hepatic fibrosis, six patients with alcoholic hepatitis, 29 patients with cirrhosis but without alcoholic hepatitis, 34 patients with both hepatic cirrhosis and alcoholic hepatitis, and four patients with alcoholic cirrhosis and hepatocellular carcinoma.

A liver biopsy specimen was obtained during the current hospital stay in the 83 alcohol abusers with abnormal liver tests. The time interval between ethane measurement and liver biopsy was less than 1 week in 75 patients. The liver histology was subsequently reviewed by one pathologist for semiquantitative grading of the following lesions: necrosis, hyaline necrosis, Mallory's bodies, microvacuolar steatosis, microvesicular steatosis, intralobular inflammation, inflammation in portal tract or in fibrosis, portal and periportal fibrosis, centrilobular fibrosis, hepatic vein lesions, iron content, cholestatis, and hepatic architecture. A global inflammation score was also calculated as the mean of the scores for intralobular inflammation and inflammation in portal tracts or fibrosis. A global hepatic alcoholic score was calculated as the mean of the various individual scores pertaining to necrosis, hyaline necrosis, Mallory's bodies, and inflammation. The following parameters were also recorded: age, sex, acknowledged daily ethanol intake before hospital admission, body temperature, ascites, encephalopathy, jaundice, oedema, mean corpuscular volume, polymorphonuclear white blood cells, serum bilirubin, serum alanine (ALT) and aspartate (AST) aminotransferase activities, alkaline phosphatase and $\gamma$-glutamyl transferase activities, serum albumin, serum IgG, plasma prothrombin level. Biological parameters were measured within 4 days of the measurement of ethane exhalation. The Pugh's score was calculated in cirrhotic patients.

\section{MEASUREMENT OF ETHANE EXHALATION}

Smokers were asked to abstain from smoking for 3 hours before the test. After explanation and acceptation of the test, the subjects were asked to first exhale normally, and then to exhale forcefully into an empty 2.5 litre gas sampling bag, thus providing alveolar air. ${ }^{7}$ Another bag was filled with ambiant air from the room to measure the concentration of ethane in the air inhaled by the subject.

The concentration of ethane in air was measured as previously reported by Lettéron et al,$^{1+}$ by gas-liquid chromatography apparatus (Packard model 437 A), equipped with a flame ionisation detector. Peak areas were integrated and calculated on a Packard recorder (model 604) with a range set at 1 and an attenuation factor set at 1 (no attenuation). Briefly, samples of air were injected through a six-way valve and a $5 \mathrm{ml}$ sample loop, in a $100 \times 0 \cdot 16 \mathrm{~cm}$ id stainless steel column packed with Carbosieve G $(60 / 80$ mesh). The column and detector temperatures were set at $170^{\circ} \mathrm{C}$ and $200^{\circ} \mathrm{C}$, respectively. The flow rate of nitrogen was set at $20 \mathrm{ml} /$ minute. Under these conditions, the retention time of ethane was about 3 minutes. Standard dilutions of pure ethane (Air Liquide, France) in air gave linear responses over a range of concentrations that exceeded both ways the range of concentrations observed in the test samples. For each test, a standard calibration was made with a dilution of ethane $(8500 \mathrm{pmol} / \mathrm{l}$ air $)$. The ethane exhaled by the subjects was calculated as the difference between the concentration of ethane in the air exhaled by the subject and that in the air inhaled (means of three injections from each gas sampling bag). The intraassay coefficient of variation was less than $10 \%$.

\section{STATISTICS}

Mean ethane exhalation in the three different groups of subjects - that is, healthy subjects, non-alcoholic patients, and alcohol abusers - was compared by one way analysis of variance followed by Dunnett's $t$ test. Univariate regression analysis was performed to assess possible relationships between ethane exhalation and the various parameters examined.

\section{Results}

Ethane exhalation was not different in the 42 healthy subjects and the 52 patients with nonalcoholic liver diseases (Fig 1). Ethane exhalation did not differ by analysis of variance among the various subgroups composing the nonalcoholic group (Table I). In contrast, the exhalation of ethane was significantly increased in the 89 alcohol abusers (Fig 1), the mean value in this group being fivefold that found in healthy subjects. The exhalation of ethane in the 89 alcohol abusers was significantly but very weakly correlated with the acknowledged daily intake of 
Figure 1: Exhalation of ethane in 42 control subjects, alcoholic liver diseases, and 89 alcohol abusers. The exhalation of ethane, mean (SEM), was assessed as the difference between the concentration of ethane in alveolar air and that in ambient air. ${ }^{\star} p<0.0001$ significantly different from that in controls. 52 patients with various non-

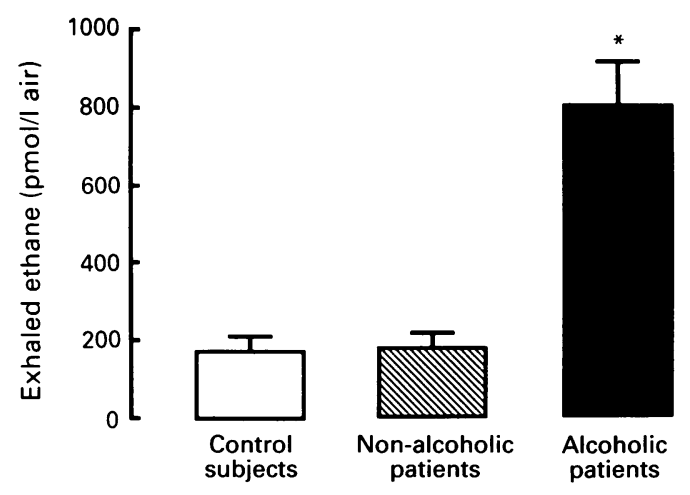

ethanol (Fig 2). It was not significantly correlated with the body temperature, the presence of ascites, encephalopathy, jaundice, or oedema, the serum bilirubin concentrations, serum albumin, or plasma prothrombin, the mean corpuscular volume, or the polymorphonuclear white blood cell counts, the activities of alkaline phosphatase or $\gamma$-glutamyl transferase, the individual values for AST or ALT activities, or the AST/ALT ratio. Ethane exhalation was significantly but very weakly correlated with the Pugh's score in the 67 alcoholic patients with liver cirrhosis (with or without alcoholic hepatitis or hepatocellular carcinoma) (Fig 2).

Analysis of variance failed to show any significant difference in the ethane exhalation among the various histological subgroups detailed in Table II. Ethane exhalation was significantly but very weakly correlated with the histological score for macrovacuolar steatosis (Fig 2) but was not correlated with any of the other liver lesions directly quantitated by the pathologist (see Methods) nor with the global inflammatory score or the global alcoholic hepatitis score.

Ethane exhalation was inversely correlated with the duration of abstinence, as assessed by the time interval between the onset of hospital admission and the first measurement of ethane exhalation (Fig 3). In nine patients, the first ethane exhalation measurement (reported in Table II) was followed by repeated measurements (Fig 3). In all of these patients, the exhalation of ethane decreased with the duration of hospital stay, and thus abstinence (Fig 3). This decrease was usually slow, however, occurring over a period of several weeks (Fig 3).

\section{Discussion}

The initial step in lipid peroxidation is the abstraction, by a drug free radical or an oxygen

TABLE I Ethane exhalation in 52 patients with various non-alcoholic liver diseases

\begin{tabular}{lc}
\hline & $\begin{array}{l}\text { Ethane exhalation } \\
\text { (pmol/l air) }\end{array}$ \\
\hline Transplanted liver $(\mathrm{n}=2)$ & 366,25 \\
Acute hepatitis $(2$ viral, 4 drug induced) & $116(26)^{\star}$ \\
Chronic hepatitis $(9$ viral, 2 autoimmune) & $70(13)^{\star}$ \\
Viral cirrhosis $(\mathrm{n}=17)$ & $228(19)^{\star}$ \\
Polyadenomatosis $(\mathrm{n}=1)$ & 149 \\
Liver carcinoma (5 hepatocellular carcinoma, 2 liver metastasis) & $95(58)^{\star}$ \\
Cholestasis (5 sclerosing cholangitis, 1 primary biliary cirrhosis, 2 extrahepatic & $159(32)^{\star}$ \\
biliary obstruction & \\
\hline
\end{tabular}

$\star$ Values are mean (SEM). radical $(\mathbf{R} \cdot)$, of a hydrogen atom from an unsaturated lipid (lipid-H), forming $\mathrm{RH}$ and a lipid radical (lipid•). This radical quickly adds molecular oxygen to form a peroxyl radical (lipid-OO.). In the presence of $\mathrm{Fe}^{2+}$, the peroxyl radical may form an alkoxyl radical $\left(\mathrm{R}_{1}-\mathrm{CHO}-\mathrm{R}_{2}\right)$, which may split into an alkyl radical $\left(\mathbf{R}_{1}{ }^{\circ}\right)$, such as the pentyl or ethyl radicals, and an aldehyde (CHO- $\left.\mathrm{R}_{2}\right)$. A hydrogen atom is abstracted by the pentyl or ethyl radical to form pentane or ethane, respectively. Pentane either is hydroxylated by hepatic cytochrome $\mathrm{P}-450$, or is exhaled in breath. ${ }^{56}$ Pentane exhalation, therefore, depends both on its formation rate and on its metabolism. ${ }^{56}$ In contrast, ethane undergoes little metabolism. Its exhalation in breath provides an index of its formation rate. ${ }^{5}$ Accordingly, exhalation of ethane is considered as a good, non-invasive, test for in vivo lipid peroxidation, particularly in patients with liver disease. $^{5}$

Our results establish, in a large series, that the average exhalation of ethane is indeed appreciably increased in alcohol abusers admitted to hospital (Fig 1). These results are consistent with several previous reports, showing changes in other markers of lipid peroxidation in chronic alcoholic patients $s^{2+1112}$ but contrast with the report of normal ethane exhalation in 15 patients with alcoholic cirrhosis. ${ }^{7}$ The reasons for this discrepancy are unknown. Five of the latter patients had been abstinent for at least 1 month before admission to hospital. ${ }^{7}$ The other patients had been drinking until admission, but the time between admission and the measurement of breath ethane was not indicated. ${ }^{7}$ This interval may have been prolonged if patients were included only after the results of other tests had been returned (such as liver pathology, ultrasonography).

At least three mechanisms whereby the metabolism of ethanol may lead to formation of free radicals and peroxidation have been proposed. ${ }^{15-21}$ These are as follows:

(a) Firstly, cytochrome P-450 partly transforms ethanol into the $\alpha$-hydroxyethyl radical $\left(\cdot \mathrm{CHOH}-\mathrm{CH}_{3}\right) .^{15-17}$

(b) Secondly, ethanol administration partly converts xanthine dehydrogenase to xanthine oxidase. ${ }^{18}$ Xanthine oxidase metabolises acetaldehyde, producing superoxide anion. ${ }^{19}$ Superoxide anion may release ferrous iron from ferritin, producing the hydroxyl radical, and initiating lipid peroxidation. ${ }^{20}$

(c) Thirdly, the metabolism of ethanol leads to reduced $\mathrm{NAD}^{+} / \mathrm{NADH}$, and $\mathrm{NADP}^{+} / \mathrm{NADPH}^{-}$ ratios. ${ }^{21}$ This may increase the microsomal reduction of ferric iron to ferrous iron, thus initiating lipid peroxidation. ${ }^{21}$

While these three mechanisms are likely to contribute to lipid peroxidation during active alcohol intoxication, they should not intervene after alcohol withdrawal. The present study shows that increased ethane exhalation still persisted after 10 days in hospital (and alcohol abstinence) in many alcohol abusers (Fig 3). Clearly, the ethanol ingested before admission to hospital would have been cleared by that time.

Increased ethane exhalation observed during the first week(s) of abstinence may, however, be 
related to relatively long lived morphological and/or biochemical lesions induced in the liver by alcohol. Because non-alcoholic liver lesions were not associated with increased ethane exhalation (Fig 1), possible causes should be sought in lesions more specifically associated with alcohol ingestion.

A major abnormality characteristically (if not exclusively) associated with alcoholism is the presence of polymorphonuclear cells in the inflammatory infiltrate. Polymorphonuclear cells are activated at sites of inflammation and release superoxide anion. ${ }^{22}$ However, they also release the iron binding protein, lactoferrin, which limits the extracellular formation of the hydroxyl radical. ${ }^{22}$ Stimulated human polymorphonuclear leukocytes do not cause peroxidation, unless iron salts are added to the medium. ${ }^{23}$ Instead, their toxicity to hepatocytes seems related to the release of proteases. ${ }^{24}$ In the present study, there was no relationship between the exhalation of ethane and the semi-quantitative assessment of the inflammatory infiltrate.

A second lesion, which is rather characteristic of alcoholic liver disease is steatosis. Fat present in the liver may serve as a substrate for lipid peroxidation. Continuing experimental studies in our laboratory clearly show that hepatic steatosis produced by ethanol and a variety of unrelated compounds (amineptine, tetracycline, various tetracycline derivatives, valproic acid, and pirprofen) is associated in each case with increased ethane exhalation in mice. In the present study, a significant relationship was observed between the exhalation of ethane and the semiquantitative estimation of macrovacuolar steatosis (Fig 2). Taken together, these experimental and human data suggest that the presence of fat deposits in liver cells may be one of the mechanisms leading to lipid peroxidation in alcohol abusers. The correlation with steatosis was very weak, albeit statistically significant cirrhosis (without or with alcoholic hepatitis or hepatocarcinoma). $r=$ correlation coefficient; $p=$ probability.
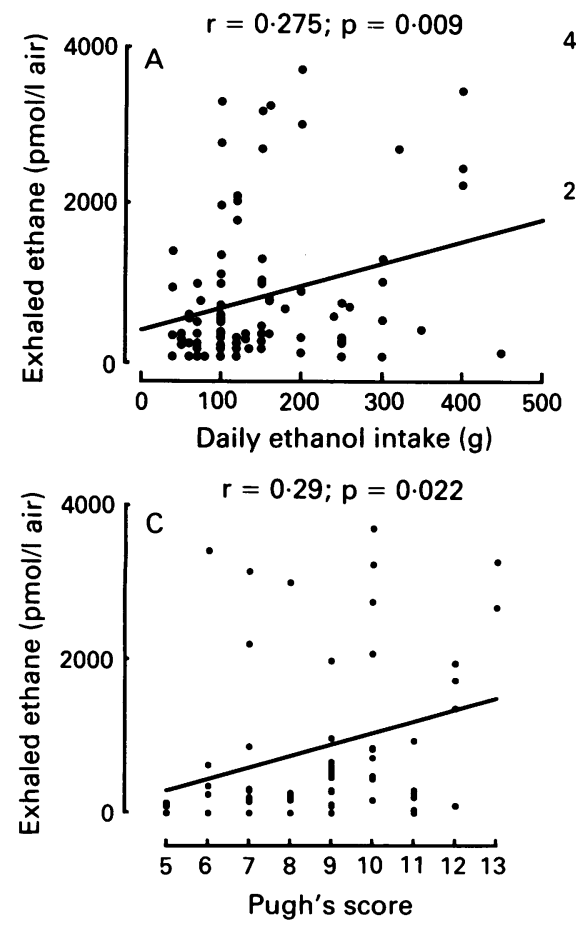

(Fig 2), however, suggesting that other factors may be involved as well.

Indeed, two biochemical modifications not assessed in our study may contribute to lipid peroxidation during the early phases of abstinence:

(a) Normally $2 \%$ of the oxygen consumed by mitochondria is transformed into superoxide anion and other reactive oxygen intermediates. ${ }^{25}$ Mitochondria from animals exposed to ethanol exhibit changes in the respiratory chain. ${ }^{26}$ Partial inhibition of the respiratory chain may cause increased oxidation of redox carriers (for example ubisemiquinone) resulting in increased production of oxygen radicals, ${ }^{27}$ and increased susceptibility to lipid peroxidation..$^{28}$

(b) Alcohol consumption induces cytochrome $\mathrm{P}-4502 \mathrm{E} 1 .{ }^{29}$ Increased activity of this isoenzyme in alcoholics may persist for several days to weeks after stopping alcohol abuse. ${ }^{29}$ Cytochrome $\mathrm{P}-4502 \mathrm{E} 1$ is peculiar by its marked ability to spontaneously form the hydroxyl radical. ${ }^{3031}$ Increased production of oxygen radicals as a consequence of mitochondrial changes and the induction of cytochrome P-450 2E1 may have contributed to increased ethane exhalation in alcohol abusers with normal liver tests (Table II). However, because a liver biopsy was not performed in these patients, the

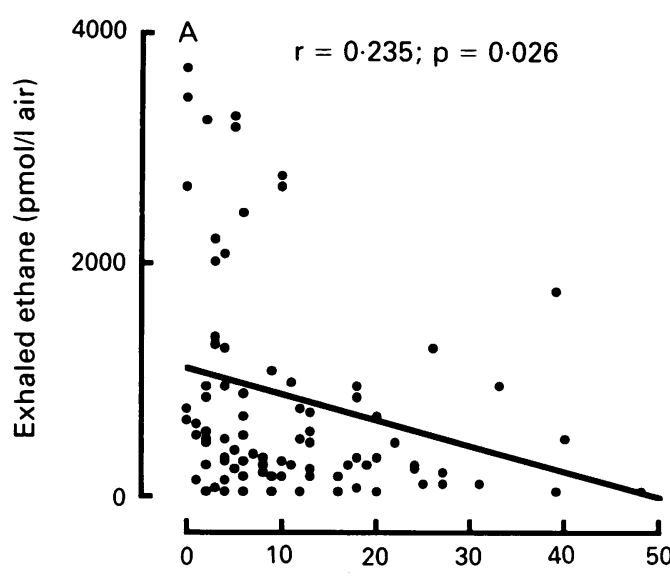

Time interval between

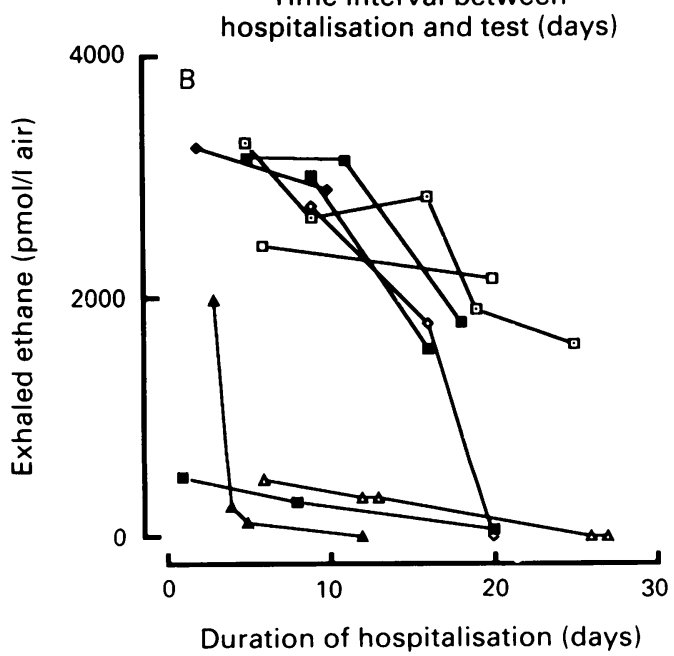

Figure 3: Inverse relationship between the exhalation of ethane, and the duration of hospital stay in the 89 alcohol abusers ( $r=$ correlation coefficient; $p=$ probability), and evolution of ethane exhalation in the nine alcohol abusers who were studied repeatedly. 
TABLE II Ethane exhalation in 89 alcohol abusers

\begin{tabular}{ll}
\hline & $\begin{array}{l}\text { Ethane exhalation } \\
(p \text { molll })\end{array}$ \\
\hline Normal liver tests $(\mathrm{n}=6)$ & $461(177)^{\star}$ \\
Steatosis, with or without fibrosis $(\mathrm{n}=10)$ & $834(227)^{\star}$ \\
Alcoholic hepatitis $(\mathrm{n}=6)$ & $450(199)^{\star}$ \\
Cirrhosis $(\mathrm{n}=29)$ & $814(207)^{\star}$ \\
Cirrhosis and alcoholic hepatitis $(\mathrm{n}=34)$ & $910(178)^{\star}$ \\
Cirrhosis and hepatocellular carcinoma $(\mathrm{n}=4)$ & $345(127)^{\star}$ \\
\hline
\end{tabular}

$\star$ Values are mean (SEM).

presence of minor liver lesions, such as steatosis, cannot be excluded.

Although the exhalation of ethane was on average increased fivefold in alcohol abusers (Fig 1), there was wide variability in this group (Table II, Fig 2). Indeed in a few alcohol abusers, there was no detectable production of ethane (that is, the ethane concentration in expired air was similar to that in inspired air). This variation may reflect both variability in alcohol consumption, in liver lesions, and in the time of measurement, as well as variability in protective mechanisms. Lipid peroxidation is efficiently prevented by vitamin $\mathrm{E}(\alpha-$ tocopherol), which transforms lipid peroxyl radicals into hydroperoxides, the latter being then transformed into lipid alcohols by glutathione peroxidase. In the process, however, $\alpha$-tocopherol is converted to the $\alpha$-tocopheroxyl radical, $\alpha$-tocopheryl quinone, and other products, ${ }^{8}$ while reduced glutathione (GSH) is oxidised to the disulphide. As a consequence of lipid peroxidation, and other factors, hepatic vitamin $E^{1112}$ and GSH levels ${ }^{2}$ are low in some alcoholics. Depletion of these protective factors may, in turn, permit the unrestrained development of lipid peroxidation in some patients.

Experimentally, lipid peroxidation has several deleterious effects on the liver. It damages membranes, including the plasma membrane, leading to increased entry of calcium into the cell, ${ }^{32}$ decreased sequestration of calcium in endoplasmic reticulum, and mitochondria, ${ }^{33} 34$ and liberation of lysosomal enzymes. ${ }^{35}$ Lipid peroxidation activates phospholipase $\mathrm{A} 2$, with the release of toxic lysophospholipids, free fatty acids, leukotrienes, and other eicosanoid metabolites. ${ }^{36}$ It forms reactive aldehydes (4hydroxyalkenals and malondialdehyde), which react with hepatic proteins. ${ }^{35}$ In the present study, however, the exhalation of ethane was similar in patients with cirrhosis and those with other liver lesions (Table II). Admittedly, progression to cirrhosis should depend not only on the instantaneous degree of a presumed pathogenic event (in this case, lipid peroxidation), but even more on the duration of this process, so that a similar instantaneous value might be seen in patients with incipient lesions and those with full blown cirrhosis. Even in the group of cirrhotic patients, however, ethane exhalation was only weakly correlated with the Pugh's score (Fig 2). This observation is consistent with the view that many other factors participate in the development of severe liver disease in these patients, including immunological mechanisms, nutritional deficiencies, mitochondrial injury, altered redox status, impaired protein processing, cyto- skeletal abnormalities, activation of Ito cells, and circulatory disturbances. ${ }^{37} 38$

In summary, the average exhalation of ethane was increased five-fold in a large hospital series of alcohol abusers, but was normal in a group of patients with various non-alcoholic liver diseases. Although the increased exhalation of ethane in alcohol abusers slowly improved after alcohol withdrawal, it was still high during the first week(s) of abstinence, suggesting that it was not related, at that time, to the production of free radicals during the metabolism of ethanol. One of the possible mechanisms may be the presence of increased amounts of oxidisable fat in the liver. The weak correlation with the Pugh's Score in alcoholic patients with cirrhosis is consistent with the contribution of many other factors in the development of severe liver disease in such patients.

1 Nordmann R, Ribière C, Rouach H. Implication of free radical mechanisms in ethanol-induced cellular injury. Free Rad Biol Med 1992; 12: 219-40

2 Shaw S, Rubin KP, Lieber CS. Depressed hepatic glutathione and increased diene conjugates in alcoholic liver disease. Evidence of lipid peroxidation. Dig Dis Sci 1983; 28: 585-9.

3 Suematsu T, Matsumara T, Sato N, Miyamoto T, Ooka T, Kamada $\mathrm{T}$, et al. Lipid peroxidation in alcoholic liver disease in humans. Alcohol Clin Exp Res 1981; 5: 427-30.

4 Mazzanti R, Moscarella S, Bensi G, Altavilla E, Gentilini P. Hepatic lipid peroxidation and aldehyde dehydrogenase activity in alcoholic and non alcoholic liver disease. Alcohol Alcohol 1989; 24: 121-8.

5 Van Gossum A, Decuyper J. Breath alkanes as an index of lipid peroxidation. Eur Respir f 1989; 2: 787-91.

6 Allerheiligen SRB, Ludden TM, Burk RF. The pharmacokinetics of pentane, a by-product of lipid peroxidation. Drug Metab Dispos 1987; 15: 794-800.

7 Moscarella S, Laffi G, Buzzelli G, Mazzanti R, Caramelli L, Gentilini P. Expired hydrocarbons in patients with chronic liver disease. Hepatogastroenterology 1984; 31: 60-3.

8 Drevon CA. Absorption, transport and metabolism of vitamin E. Free Rad Res Commun 1991; 14: 229-46.

9 Sokol RJ. Vitamin E and neurologic function in man. Free Rad Biol Med 1989; 6: 189-207.

10 Sokol RJ, Kim YS, Hoofnagle JH, Heubi JE, Jones EA, Balistreri WF. Intestinal malabsorption of vitamin $\mathrm{E}$ in primary biliary cirrhosis. Gastroenterology 1989;96: 479-86.

11 Tanner AR, Bantock I, Himks L, Lloyd B, Turner NR Wright R. Depressed selenium and vitamin E levels in an alcoholic population. Possible relationship to hepatic injury through increased lipid peroxidation. Dig Dis Sci 1986; 31 1307-12.

12 Girre C, Hispard E, Therond P, Guedj S, Bourdon R, Dally S Effect of abstinence from alcohol on the depression of glutathione peroxidase activity and selenium and vitamin $\mathrm{E}$ levels in chronic alcoholic patients. Alcohol Clin Exp Res 1990; 14: 909-12.

13 Jeffrey GP, Muller DPR, Burroughs AK, Matthews S, Kemp $\mathrm{C}$, Epstein $\mathrm{O}$, et al. Vitamin $\mathrm{E}$ deficiency and its clinical significance in adults with primary biliary cirrhosis and other significance in adults with primary biliary cirrhosis and other

14 forms of chronic liver disease. F Hepatol 1987; 4: 307-17. Delaforge $\mathrm{M}$, et al. Mechanism for the protective effects of silymarin against carbon tetrachloride-induced lipid peroxidation in mice. Evidence that silymarin acts both as an inhibitor of metabolic activation and as a chain-breaking antioxidant. Biochem Pharmacol 1990; 39: 2027-34

15 Albano E, Tomasi A, Goria-Gatti L, Dianzani MU. Spin trapping of free radical species produced during the microsomal metabolism of ethanol. Chem Biol Interact 1988; 65 . 223-34.

16 Reinke LA, Lai EK, Dubose CM, McCay PB. Reactive free radical generation in vivo in heart and liver of ethanol-fed rats: correlation with radical formation in vitro. Proc Natl Acad Sci USA 1987; 84: 9223-7.

17 Knecht KT, Bradford BU, Mason RP, Thurman RG. In vivo formation of a free radical metabolite of ethanol. Mol formation of a free radical

18 Sultatos LG. Effects of acute ethanol administration on the hepatic xanthine dehydrogenase/oxidase system in the rat. 7 Pharmacol Exp Ther 1988; 246: 946-9.

19 Shaw S, Jayatilleke E. The role of aldehyde oxidase in ethanolinduced hepatic lipid peroxidation in the rat. Biochem $f$ 1990; 268: 579-83.

20 Biemond P, Swaak JG, Beindorf CM, Koster JF. Superoxidedependent and -independent mechanisms of iron mobilization from ferritin by xanthine oxidase. Biochem $f$ 1986; 239: 169-73.

21 Kukielka E, Cederbaum AI. NADH-dependent microsomal interaction with ferric complexes and production of reactive oxygen intermediates. Arch Biochem Biophys 1989; 275: $540-50$ 
22 Britigan BE, Rosen GM, Thompson BY, Chai Y, Cohen MS Stimulated human neutrophils limit iron-catalyzed hydroxyl radical formation as detected by spin-trapping techniques. Biol Chem 1986; 261: 17026-32.

23 Thomas MJ, Shirley PS, Hedrick CC, DeChatelet LR. Role of free radical processes in stimulated human polymorphonuclear leukocytes. Biochemistry 1986; 25: 8042-8.

24 Mavier P, Preaux AM, Guigui B, Lescs MC, Zafrani ES Dhumeaux D. In vitro toxicity of polymorphonuclear neutrophils to rat hepatocytes: Evidence for a proteinasemediated mechanism. Hepatolog $v 1988 ; 8: 254-8$.

25 Boveris A, Oshino N, Chance B. The cellular production of hydrogen peroxide. Biochem 7 1972; 128: 617-30.

26 Spach PI, Cunningham CC. Control of state 3 respiration in liver mitochondria from rats subjected to chronic ethanol consumption. Biochim Biophys Acta 1987; 894: 460-7.

27 Cederbaum AI. Introduction: role of lipid peroxidation and oxidative stress in alcohol toxicity. Free Rad Biol Med 1989; oxidative

28 Rouach H, Clement M, Orfanelli MT, Janvier B, Nordman J, Nordmann R. Hepatic lipid peroxidation and mitochondrial susceptibility to peroxidative attacks durin ethanol inhalation and withdrawal. Biochim Biophys Acta 1983; 753: 439-44.

29 Lieber CS. Interaction of alcohol with other drugs and nutrients. Implication disease. Drugs 1990; 40 (suppl 3): 23-44.

30 Krikun G, Lieber CS, Cederbaum AI. Increased microsomal oxidation of ethanol by cytochrome P-450 and hydroxyl radical-dependent pathways after chronic ethanol consumption. Biochem Pharmacol 1984; 33: 3306-9.

31 Persson JO, Terelius Y, Ingelman-Sundberg M. Cytochrome P-450-dependent formation of reactive oxygen radicals: isozyme-specific inhibition of P-450-mediated reduction of oxygen and carbon tetrachloride. Xenobiotica 1990; 20: 887900

32 Lauterburg BH. Early disturbance of calcium translocation across the plasma membrane in toxic liver injury. Hepatology 1987; 7: 1179-83.

33 Massini A, Trenti T, Ventura E, Ceccarelli D, Muscatello U. The effect of ferric iron complex on $\mathrm{Ca}^{2}$ transport in isolated rat liver mitochondria. Biochem Biophys Res Commun 1985; 130: 207-13.

34 Pencil SD, Glende EA, Recknagel RO. Loss of calcium sequestration capacity in endoplasmic reticulum of isolated sequestration capacity in endoplasmic reticulum of isolated hepatocytes treated with carbon tetrachlor

35 Younes M, Siegers CP. Interelation between lipid peroxidation and other hepatotoxic events. Biochem Pharmaco 1984; 33: 2001-3.

36 Borowitz SM, Montgomery C. The role of phospholipase A2 in microsomal lipid peroxidation induced with $t$-butyl hydroperoxide. Biochem Biophys Res Commun 1989; 158: 1021-8.

37 Lieber CS. Biochemical and molecular basis of alcoholicinduced injury to liver and other tissues. N Englf Med 1988; 319: 1639-50.

38 Lieber CS. Mechanism of ethanol induced hepatic injury. Pharmacol Ther 1990; 46: 1-41. 